\title{
Mitigating modulation instability in Brillouin distributed fibre sensors
}

\author{
Marcelo A. Soto*, Mehdi Alem, Wei Chen ${ }^{\dagger}$, Luc Thévenaz \\ EPFL Swiss Federal Institute of Technology, Institute of Electrical Engineering, \\ SCI STI LT, Station 11, CH-1015 Lausanne, Switzerland; \\ 'Permanent address: College of Optoelectronic Science and Engineering, \\ National University of Defense Technology, Changsha 410073, China; \\ *E-mail: marcelo.soto@epfl.ch
}

\begin{abstract}
A thorough study of the generation of modulation instability (MI) in distributed Brillouin fibre sensors is presented. ASE noise co-propagating with the pump pulses within the MI gain spectrum has been identified to be an important factor seeding modulation instability and reducing its power threshold. The paper describes how optical narrowband filtering reduces the pump depletion resulting from MI, allowing pump pulses to propagate through longer distances in standard optical single-mode fibres.
\end{abstract}

Keywords: Optical fibre sensor, stimulated Brillouin scattering, modulation instability, distributed fibre sensor

\section{INTRODUCTION}

Over the past few years, extensive research on Brillouin optical time-domain analysis (BOTDA) for distributed strain and temperature fibre sensing has been carried out, focussing mainly on high spatial resolution and long sensing range. For long-distance sensing, high optical power could be used to compensate the cumulated fibre loss and to obtain a high enough signal-to-noise ratio (SNR) at the most remote sensing point. Unfortunately, fibre nonlinearities also turn significant under such conditions and only a limited amount of optical power can therefore be launched into the sensing fibre $^{1}$. In particular, the continuous-wave probe power cannot be increased beyond the threshold of stimulated Brillouin scattering (SBS), which moreover induces pump depletion and leads to systematic errors in Brillouin frequency shift (BFS) measurements ${ }^{2}$. On the other hand, the maximum pump power that can be allowed inside the fibre is limited by modulation instability (MI) and stimulated Raman scattering (SRS), which might introduce considerable pump depletion, limiting then the maximum sensing range ${ }^{1}$. Compared with SRS, MI has a lower threshold and it can be suppressed using fibres with normal dispersion ${ }^{1}$. However, for standard single-mode fibres (SMF), commonly used in real BOTDA systems, MI inevitably plays an important role due to the anomalous dispersion ${ }^{1,3}$. This way, the most limiting factor to extend the sensing distance of BOTDA sensors has been found to be the onset of nonlinear effects, and in particular $\mathrm{MI}^{3}$.

In this paper the generation of modulation instability in real BOTDA systems is analysed. It is shown how amplified spontaneous emission (ASE) noise, resulting from the erbium-doped fibre amplifier (EDFA) typically employed to boost the peak pump power, can seed modulation instability even with low pump power. Considering that the MI gain spectrum is about some few tens of GHz in standard SMFs, the impact of MI on BOTDA measurements is analysed when narrowband optical filtering is used to reduce the ASE noise from the pump wave.

\section{INITIALISATION OF MODULATION INSTABILITY IN BOTDA SENSORS}

Modulation instability has detrimental effects on both Brillouin optical time-domain reflectometry (BOTDR $)^{4}$ and BOTDA $^{3}$ optical fibre sensors. In BOTDR sensors, based on spontaneous Brillouin scattering, in addition to pump depletion, MI contaminates the detected Brillouin signal so that there is an upward drift in the BFS leading to an upward drift in the measured temperature/strain along the fibre ${ }^{4}$. On the other hand, for BOTDA sensors the pump depletion due to $\mathrm{MI}$ is more critical since the SBS gain depends on the pump power ${ }^{1,3}$. Modulation instability can occur with lower pump power in BOTDA systems compared to BOTDR sensors because the Rayleigh backscattering of the probe signal, typically lying within the MI frequency band, can seed the MI process.

Modulation instability in optical fibres results from the interplay between the group velocity dispersion (GVD) in anomalous regime $\left(\beta_{2}<0\right)$ and the nonlinear Kerr effect represented by the nonlinear coefficient $\gamma$. Using the linear

Fifth European Workshop on Optical Fibre Sensors, edited by Leszek R. Jaroszewicz, Proc. of SPIE Vol. 8794, 87943J · @ 2013 SPIE · CCC code: 0277-786X/13/\$18 · doi: 10.1117/12.2026296 
stability analysis of the nonlinear Schrodinger (NLS) equation ${ }^{5}$ for a lossless fibre in undepleted pump regime, it can be shown that the spectral distribution of the modulation instability gain is:

$$
g(\Omega)=1+2 \frac{\left(\frac{2 \gamma P}{\beta_{2}}\right)^{2} \sinh ^{2}\left(\frac{\beta_{2} L}{2} \sqrt{\Omega^{2}\left(\Omega_{\mathrm{c}}{ }^{2}-\Omega^{2}\right)}\right)}{\Omega^{2}\left(\Omega_{\mathrm{c}}{ }^{2}-\Omega^{2}\right)},
$$

in which $P$ is the pump power and $\Omega_{c}=\sqrt{4 \gamma P /\left|\beta_{2}\right|}$ determines the MI frequency band, where $\Omega$ is the detuning from the pump frequency. The maximum MI gain occurs at the frequencies $\pm \Omega_{\mathrm{c}} / \sqrt{2}$ that depends on the dispersion $\beta_{2}$, and peaks to $1+2 \sinh ^{2}(\gamma L P)$, which is independent of dispersion. Fig.1 depicts the MI gain in an SMF, obtained from Eq. (1) and also by simulating the NLS equation using split-step Fourier transform ${ }^{5}$. It can immediately be pointed out the MI gain band matches fairly well the spectral range where signals interacting through SBS are present.

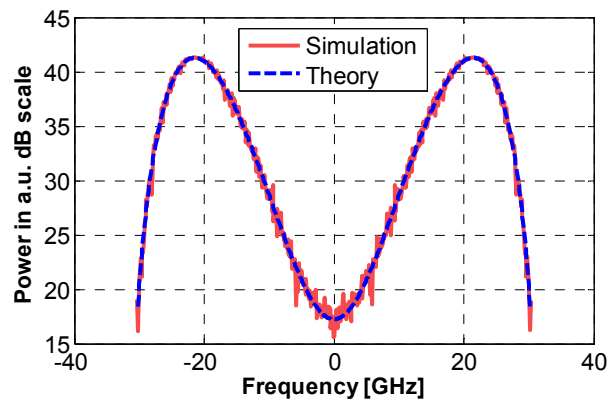

Figure 1. MI gain spectrum for a $25 \mathrm{~km} \mathrm{SMF} \mathrm{using} 100 \mathrm{~mW}$ input power and typical values of $\beta_{2}=-22 \mathrm{ps}^{2} / \mathrm{nm}$ and $\gamma=1.8(\mathrm{kmW})^{-1}$.

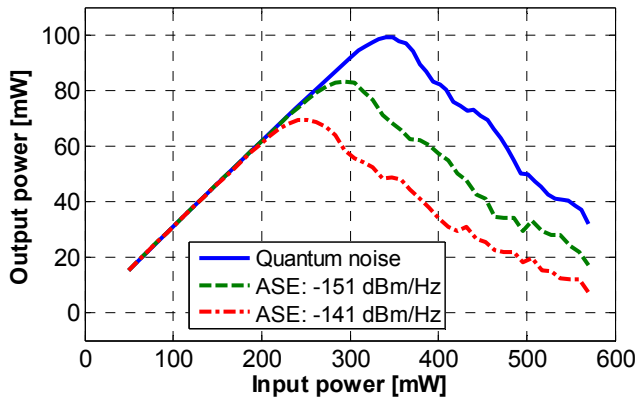

Figure 2. Output versus input pump power for different noise levels in a $25 \mathrm{~km}$ SMF showing the effect of noise on pump depletion due to MI.

Modulation instability starts from the background noise in the MI frequency band, and therefore, noise co-propagating with the pump in a BOTDA system can easily seed MI, resulting in pump depletion mainly at long distances. In BOTDA sensors the pump power is usually boosted by an EDFA. Therefore, depending on the input pulse power and pulse repetition rate (given by the fibre length), the EDFA can operate in linear or saturated regime, so that any possible level of ASE noise should be expected at the fibre input. In particular, the amplification of optical pulses at low-repetition rates using an EDFA results in high levels of ASE noise, which also depends on the EDFA pumping conditions. Fig. 2 shows the output versus the input pump power of an SMF for different levels of white background noise. Results have been obtained by numerically solving the NLS equation ${ }^{5}$, assuming a $25 \mathrm{~km} \mathrm{SMF}$ with an attenuation of $0.2 \mathrm{~dB} / \mathrm{km}$. It is possible to observe a first linear regime in which the output power increases with the input power accordingly, and a second region, under pump depletion conditions, in which not only the output power reduces while increasing the input power, but it also exhibits an oscillatory behaviour due to the so-called Fermi-Pasta-Ulam (FPU) recurrence ${ }^{1}$. Results shown in Fig. 2 actually point out that due to 'quantum noise' it is practically impossible to completely cancel out MI in optical fibres with anomalous dispersion. However, it can be easily seen that by decreasing the level of ASE noise in the fibre the power threshold of modulation instability can be increased, so that pump depletion due to MI could only occur at substantially higher pump power levels. Therefore, by using narrowband optical filtering, the ASE noise within the MI frequency band (presenting a maximum gain at $\sim 20 \mathrm{GHz}$, as shown in Fig. 1, for standard BOTDA conditions) can be reduced, mitigating this way the pump depletion resulting from MI.

\section{VALIDATING SETUP}

The validating setup is sketched in Fig. 3. The scheme is practically similar to a standard BOTDA configuration, in which the light from a distributed feedback (DFB) laser diode (LD) at $\sim 1551 \mathrm{~nm}$ is coupled into the pump and probe branches by a 50:50 coupler. For the pump branch, a semiconductor optical amplifier (SOA) is used for generating pulses with high extinction ratio. Pulses are amplified by an EDFA and attenuated by a variable optical attenuator (VOA) to precisely adjust different pump peak powers. In the dashed box, a circulator and a fibre Bragg grating (FBG) with $1 \mathrm{GHz}$ bandwidth are used to filter out the ASE noise from the pulsed pump; however, those devices have also been removed during measurements for comparison purposes. Then, a 1:99 coupler is inserted to perform a calibrated monitoring of the input pump power launched into a $25 \mathrm{~km}$ standard SMF. For the probe branch, an electro-optic modulator (EOM) is used to generate a two-sideband probe with suppressed carrier, and a polarisation switch is inserted to eliminate the polarisation dependence on the SBS gain. Before the photo-detector (PD), a 10-GHz FBG transmits only one probe sideband to the detection stage. Finally time-domain traces are recorded using a computer acquisition card. 


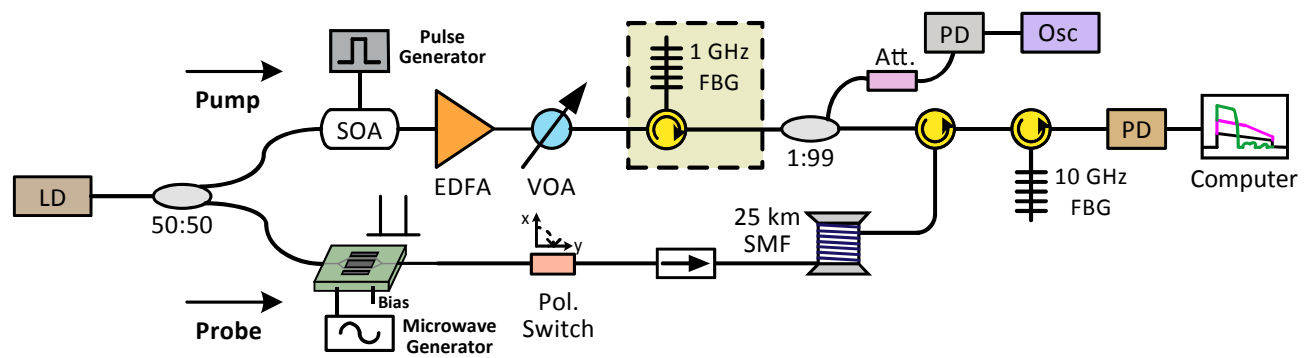

Figure 3. Experimental setup. LD: laser diode; SOA: semiconductor optical amplifier; EDFA: erbium-doped fibre amplifier; VOA: variable optical attenuator; FBG: fibre Bragg grating; Att: $10 \mathrm{~dB}$ attenuator; PD: photodetector; Osc: Oscilloscope; EOM: electric-optic modulator; SMF: single-mode fibre.

\section{EXPERIMENTAL RESULTS}

The first set of measurements is carried out to evaluate the impact of MI on BOTDA measurements when different levels of ASE noise are launched into the sensing fibre under constant pump power conditions. A proof-of-concept experiment has been first designed based on the setup shown in Fig. 3, in which considerable levels of ASE noise have been introduced co-propagating with the pump pulses. This has been realised by replacing the 1-GHz FBG (inside the dashed box) by a coupler and a low-power EDFA with no input signal. Two pump power conditions have been measured while changing the ASE power spectrum continuously between $-135 \mathrm{dBm} / \mathrm{Hz}$ and $-115 \mathrm{dBm} / \mathrm{Hz}$ : the first one corresponds to a low power level $(60 \mathrm{~mW})$ at which $\mathrm{MI}$ is not expected to occur, while in the second case MI is made flagrantly visible using a high pump power $(500 \mathrm{~mW})$. Measurements have been acquired with 2000 time-averaged traces using $2 \mathrm{~m}$ spatial resolution. Fig. 4(a) shows the case of $60 \mathrm{~mW}$ pump power; it is possible to observe that under the lowest noise situation, the time-domain BOTDA trace (blue curve) at the peak Brillouin gain can be measured with no distortion. However, when the ASE noise increases, MI is seeded enough to make the pump depleted. This demonstrates that the onset of modulation instability in a BOTDA sensor not only depends on the peak pump power, as previously reported in the literature ${ }^{1,3}$, but it also depends on the power of ASE noise co-propagating with the pump within the MI frequency band. Fig. 4(b) reports similar results when $500 \mathrm{~mW}$ are used. In this case MI distorts the BOTDA traces even at low noise conditions; however, the presence of ASE noise further seeds MI, increasing the amount of pump depletion.
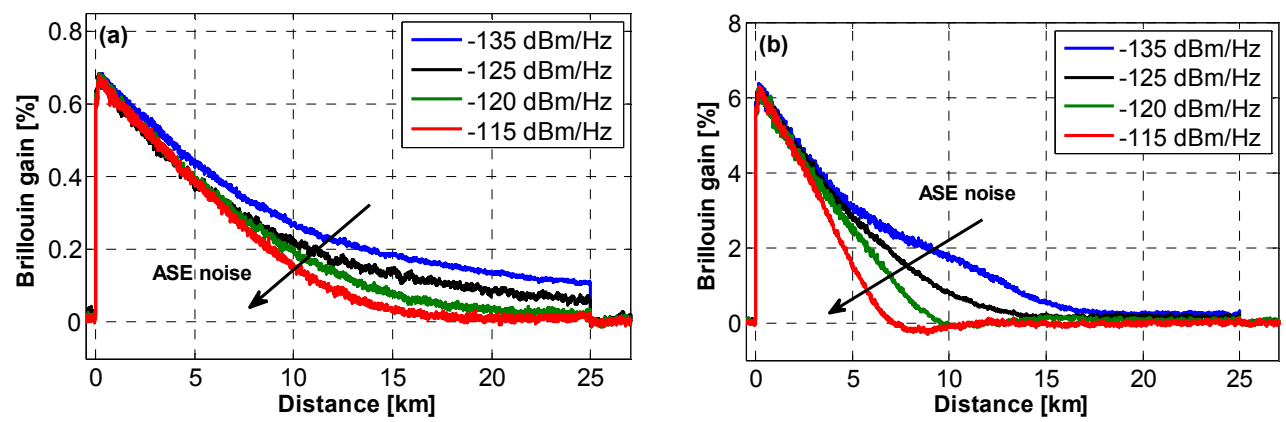

Figure 4. BOTDA traces versus distance (peak Brillouin gain) at different ASE noise power levels, for a constant peak pump power of (a) $60 \mathrm{~mW}$ and (b) $500 \mathrm{~mW}$.

Then, time-domain traces have been measured using the standard BOTDA configuration described in Fig. 3, under different pump power conditions. To evaluate the impact of the ASE noise, measurements with and without narrowband filtering have been compared. Fig. 5(a) illustrates the impact of MI under standard conditions (i.e. without filter). While a pump power of $130 \mathrm{~mW}$ allows measuring up to the far fibre end $(25 \mathrm{~km})$, increasing the peak pump power actually reduces considerably the maximum sensing range. On the other hand, under similar pump power conditions, but introducing a $1 \mathrm{GHz}$ FBG into the system (see Fig, 3), Fig. 5(b) shows that the pump can propagate for longer distances before being completely depleted. This demonstrates that narrowband filtering reduces the seeding of MI, so that pump powers up to $450 \mathrm{~mW}$ still allow measuring considerable levels of Brillouin gain at the farthest sensing point. This is also illustrated in Fig. 5(c), which shows the Brillouin gain at $25 \mathrm{~km}$ as a function of the input power with and without optical filter. The figure also reports the expected behaviour in case of narrowband filtering, obtained by solving the NLS equation for MI using split-step Fourier transform ${ }^{5}$. It is clearly seen that the use of the narrowband filter increases the threshold of modulation instability, so that higher pump powers can be launched into the fibre before the pump seriously 

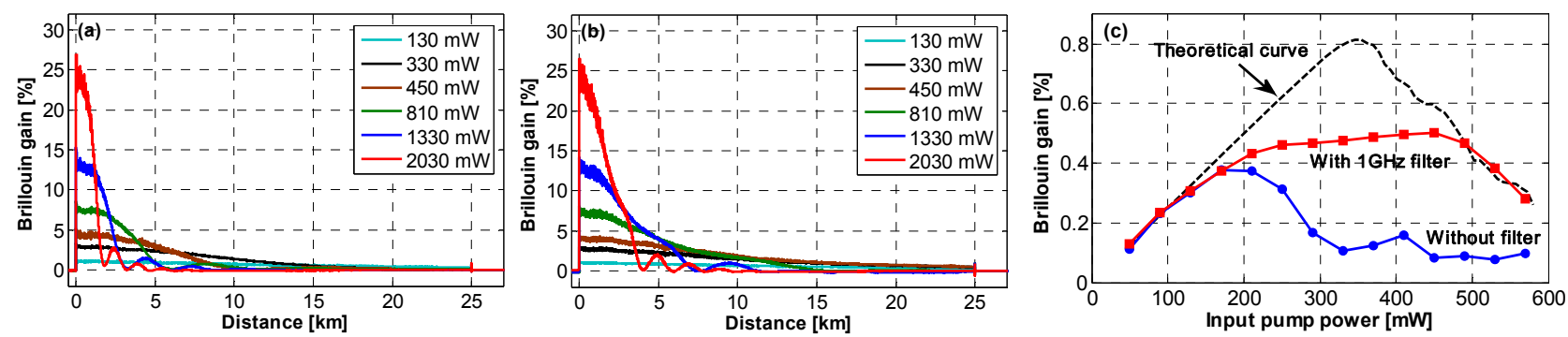

Figure 5. BOTDA traces versus distance (peak Brillouin gain) at different pump power levels. (a) Standard BOTDA conditions, and (b) with narrowband optical filter. (c) SBS gain at $25 \mathrm{~km}$ versus input pump power, with (red squares) and without (blue circles) $1 \mathrm{GHz}$ filter. Black dashed line: expected behaviour for the case of narrow filtering.

depletes. It is believed that the spectral content of the remaining noise might partially explain the differences between expected and measured curves. However, other phenomena affecting the MI evolution, such as the Rayleigh backscattered light from the probe, are still under investigation. Results shown in Fig. 5 have also been compared with the case of standard band-pass optical filtering, with bandwidths in the range of $0.5-1.0 \mathrm{~nm}$; however, no appreciable differences have been observed when compared to the non-filtered case. This is because MI extends over a band of $\sim 60 \mathrm{GHz}$, and any optical filter broader than the MI band will therefore have a negligible impact on reducing modulation instability.

Finally, Fig. 6 shows an experimental comparison of the maximum distance that the pump power can reach before being depleted by $50 \%$. It can be observed that for fibres shorter than the nonlinear effective length $\left(\alpha^{-1}=21 \mathrm{~km}\right)$, a sensing range extension equal to a factor of approximately 2 has been achieved using the $1 \mathrm{GHz}$ filter. On the other hand, for long-range sensing (longer than $\alpha^{-1}=21 \mathrm{~km}$ ), results indicate that more than $400 \mathrm{~mW}$ can be launched into the fibre before significant pump depletion occurs. Considering that the analysed $25 \mathrm{~km}$ fibre is longer than the nonlinear effective length, additional modulation instability is not expected to substantially occur when increasing the sensing range. However, it is not clear yet which amount of pump depletion the system can tolerate for stable operation. Actually, a further study remains necessary to define an appropriate MI threshold power in BOTDA sensors considering the seeding provided by the EDFA ASE noise and the Rayleigh scattering from the probe signal.

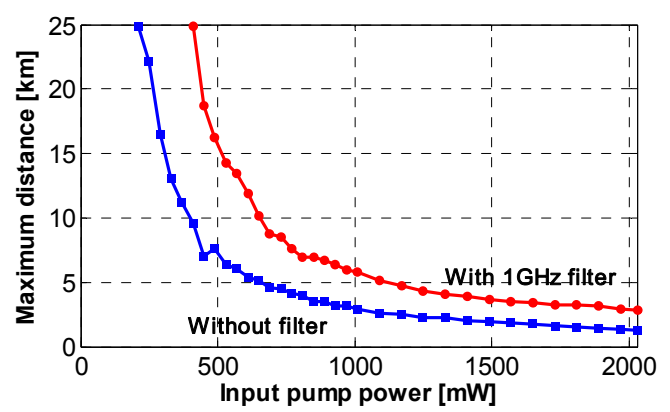

Figure 5. Maximum distance reached by the pump before $50 \%$ of its power is depleted. Comparison for both cases: with and without $1 \mathrm{GHz}$ filter.

In conclusion a thorough analysis of the generation of modulation instability in BOTDA systems has been carried out. The detrimental impact of ASE noise co-propagating with the pump and seeding MI has been theoretically and experimentally evaluated. It was demonstated that the threshold power for MI does not only depend on the pump power but also on the ASE noise co-propagating within the MI gain spectrum. While such an issue has to be considered in future mathematical formulations of the MI threshold in BOTDA sensors, narrowband filtering can mitigate pump depletion and has the potential to extend the sensing distance for short- and long-range applications.

The authors would like to acknowledge the support from the company OMNISENS (Switzerland) and from the Swiss Commission for Technology and Innovation (Project 13122.1).

\section{REFERENCES}

[1] Foaleng, S. M., and Thévenaz, L., "Impact of Raman scattering and modulation instability on the performances of Brillouin sensors," Proc. of SPIE, vol. 7753, 77539V (2011).

[2] Thévenaz, L., Foaleng, S. and Lin, J., "Impact of pump depletion on the determination of the Brillouin gain frequency in distributed fiber sensors," Proc. of SPIE, vol. 7753, 775322 (2011).

[3] Alasia, D. et al., "Detrimental effect of modulation instability on distributed optical fiber sensors using stimulated Brillouin scattering," Proc. of SPIE, vol. 5855, 587-590 (2005).

[4] Alahbabi, M. N., Cho, Y. T., and Newson, T. P., "Influence of modulation instability on distributed optical fiber sensors based on spontaneous Brillouin scattering," J. Opt. Soc. Am. B 21(6), 1156-1160 (2004).

[5] Agrawal, G. P., Nonlinear Fiber Optics, $4^{\text {th }}$ ed., Academic Press (2007). 